CLINICAL RESEARCH ARTICLE

\title{
Body composition of extremely preterm infants fed protein- enriched, fortified milk: a randomized trial
}

\author{
Ariel A. Salas ${ }^{1 凶}$, Maggie Jerome ${ }^{2}$, Amber Finck ${ }^{1}$, Jacqueline Razzaghy ${ }^{1}$, Paula Chandler-Laney ${ }^{2}$ and Waldemar A. Carlo ${ }^{1}$ \\ (c) The Author(s), under exclusive licence to the International Pediatric Research Foundation, Inc 2021
}

BACKGROUND: Critically ill extremely preterm infants fed human milk are often underrepresented in neonatal nutrition trials aimed to determine the effects of enteral protein supplementation on body composition outcomes.

METHODS: Masked randomized trial in which 56 extremely preterm infants 25-28 weeks of gestation were randomized to receive either fortified milk enriched with a fixed amount of extensively hydrolyzed protein (high protein group) or fortified milk without additional protein (standard protein group).

RESULTS: Baseline characteristics were similar between groups. In a longitudinal analysis, the mean percent body fat (\%BF) at 30-32 weeks of postmenstrual age (PMA), 36 weeks PMA, and 3 months of corrected age (CA) did not differ between groups (17 \pm 3 vs. $15 \pm 4 ; p=0.09)$. The high protein group had higher weight $(-0.1 \pm 1.2$ vs. $-0.8 \pm 1.3 ; p=0.03)$ and length $(-0.8 \pm 1.3$ vs. $-1.5 \pm$ $1.3 ; p=0.02) z$ scores from birth to 3 months CA. The high protein group also had higher fat-free mass (FFM) $z$ scores at 36 weeks PMA $(-0.9 \pm 1.1$ vs. $-1.5 \pm 1.1 ; p=0.04)$.

CONCLUSIONS: Increased enteral intake of protein increased FFM accretion, weight, and length in extremely preterm infants receiving protein-enriched, fortified human milk.

Pediatric Research (2022) 91:1231-1237; https://doi.org/10.1038/s41390-021-01628-x

\section{IMPACT:}

- Extremely preterm infants are at high risk of developing postnatal growth failure, particularly when they have low fat-free mass gains.

- Protein supplementation increases fat-free mass accretion in infants, but several neonatal nutrition trials aimed to determine the effects of enteral protein supplementation on body composition outcomes have systematically excluded critically ill extremely preterm infants fed human milk exclusively.

- In extremely preterm infants fed fortified human milk, higher enteral protein intake increases fat-free mass accretion and promotes growth without causing excessive body fat accretion.

\section{INTRODUCTION}

With the steady decrease in mortality rates among extremely preterm infants ( 28 weeks of gestation or less), ${ }^{1}$ the risk of several morbidities, including postnatal growth faltering attributed to slow weight gain, has increased in this vulnerable population. ${ }^{2}$ While numerous randomized trials have determined the quantitative effects of hospital-based nutritional interventions on weight gain, ${ }^{3,4}$ only a limited number of randomized trials have determined the qualitative effects of these interventions on body composition, specifically fat mass (FM) and fat-free mass (FFM) accretion in extremely preterm infants. 5

Weight gain resulting from FFM gains increases brain size ${ }^{7}$ and reduces the risk of adverse neurodevelopmental outcomes among preterm infants. ${ }^{8}$ Conversely, weight gain resulting from excessive FM gains could increase the susceptibility to metabolic reprogramming $^{9}$ and the risk of adverse metabolic outcomes. ${ }^{10,11}$ This evidence suggests that any nutritional intervention that reduces the risk of postnatal growth faltering should ideally improve the quantitative (i.e., weight, length, and head circumference) and qualitative (i.e., body composition) outcomes of growth. ${ }^{12,13}$ Body composition measurements are not part of routine clinical care, but reference values for preterm infants have been recently defined. ${ }^{14}$

Because muscle, bone, brain, and other structures that account for FFM are built on a protein matrix, ${ }^{15}$ higher protein intake could increase weight gain, minimize FM gains, and maximize FFM gains. ${ }^{16}$ These potential benefits are critical for extremely preterm infants fed human milk. They often receive fortified human milk that contains low and variable amounts of protein. ${ }^{17}$ More evidence is needed to support current protein supplementation practices in these high-risk infants. We hypothesized that adding a fixed amount of protein to fortified human milk reduces percent body fat $(\% \mathrm{BF})$ and increases FFM accretion in extremely preterm infants.

${ }^{1}$ Department of Pediatrics, University of Alabama at Birmingham, Birmingham, AL, USA. ${ }^{2}$ Department of Nutrition Sciences, University of Alabama at Birmingham, Birmingham,

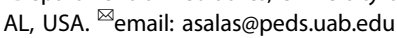




\section{METHODS}

\section{Trial design}

This study was a parallel-group, masked, randomized clinical trial in which extremely preterm infants were randomly assigned to receive either high or standard protein supplementation with a 1:1 allocation ratio. The study protocol was approved by the University of Alabama at Birmingham Institutional Review Board. Written parental consent was obtained within the first two weeks after birth. Patient recruitment began in July 2018 and continued until December 2019. Follow-up visits for body composition measurements at 3 months of corrected age (CA) began in February 2019 and ended abruptly in March 2020 due to the SARS-CoV-2 pandemic. Because it was impossible to complete follow-up visits in a considerable proportion of infants, the trial registration was updated and the original primary outcome of mean $\% \mathrm{BF}$ at 3 months CA was replaced by the outcome of mean \%BF between 30 and 32 weeks of postmenstrual age (PMA) and 3 months CA before data analysis.

\section{Participants}

Extremely preterm infants with gestational ages between 25 and 28 weeks of gestation were included. Infants with gastrointestinal malformations, central nervous system malformations, or terminal illness were excluded.

\section{Randomization, allocation concealment, and masking}

A computer-generated, random-block sequence was used to randomize study participants. Numbered, opaque, sealed envelopes were opened in sequential order to allocate the study intervention. Twin infants were randomized individually. The study intervention was masked.

\section{Interventions}

Infants fed maternal or donor milk fortified with a commercially available human milk fortifier containing casein hydrolysate (Similac Human Milk Fortifier Hydrolyzed Protein Concentrated Liquid, Abbott, Columbus, $\mathrm{OH}$ ) were randomly assigned in a 1:1 allocation ratio to receive either fortified human milk enriched with a fixed amount of a commercially available protein supplement containing casein hydrolysate (high protein group) or fortified human milk without the additional protein supplement (standard protein group). In the high protein group, extensively hydrolyzed liquid protein (Liquid Protein Fortifier, Abbott, Columbus, $\mathrm{OH}$ ) was added to fortified human milk to increase the protein content of human milk by $\sim 0.75 \mathrm{~g}$ per $100 \mathrm{ml}$.

\section{Primary and secondary outcomes}

The revised primary outcome was \%BF estimated between 30 and 32 weeks PMA and 3 months CA. Other pre-specified, secondary efficacy outcomes were anthropometric measurements (weight, head circumference, and length) from birth to 3 months CA; FM, FFM, \%BF at 36 weeks PMA or hospital discharge (whichever occurred first); postnatal growth failure (below 10th percentile) at 36 weeks PMA or discharge, and growth rate in $\mathrm{g} / \mathrm{kg} /$ day from birth to 36 weeks PMA estimated by using the exponential method. ${ }^{18}$ Safety outcomes included death, necrotizing enterocolitis (NEC) stage 2 or greater, metabolic acidosis, and uremia.

\section{Study procedures}

The study intervention began the day after standard bovine fortification was initiated and continued until 32 weeks PMA or postnatal day 50, whichever occurred first. Body composition was measured with airdisplacement plethysmography (ADP) at $\sim 30-32$ weeks PMA, at 36 weeks PMA or discharge, and at 3 months CA. For inpatient body composition measurements, infants were transported from the neonatal unit to an exam room if they were no longer requiring respiratory support with mechanical ventilation, CPAP or HFNC. For outpatient body composition measurements at 3 months CA, a follow-up visit was scheduled. All these body composition measurements were converted into $z$ score values using updated, sex-specific, reference curves of body composition in preterm infants. ${ }^{14}$ Anthropometric measurements at birth, postnatal day 7 , postnatal day 14, postnatal day 28, 32 weeks PMA, and 36 weeks PMA or discharge were converted into $z$ score values using the INTERGROWTH21 st growth curves. ${ }^{19}$ Individual protein and energy intakes were calculated after study completion assuming that the energy content of human milk was $20 \mathrm{kcal} / \mathrm{oz}(0.67 \mathrm{kcal} / \mathrm{ml})$.

\section{Control or monitoring of co-interventions}

During the trial, enteral nutrition was initiated at $20-25 \mathrm{ml} / \mathrm{kg} /$ day for $1-5$ days and then advanced by $20-25 \mathrm{ml} / \mathrm{kg} /$ day until full enteral nutrition was established (>150 ml/kg/day). Bovine-based fortifiers were added to human milk when enteral nutrition reached volumes greater than $120 \mathrm{ml} /$ $\mathrm{kg} /$ day. If the supply of human milk was insufficient to advance and continue enteral nutrition, donor milk was offered until 32 weeks PMA. If the supply of human milk was insufficient after 32 weeks PMA, the preterm formula was prescribed until discharge.

\section{Sample size}

A sample size of 56 infants was considered sufficient to detect a 3 percentage points difference in a $t$-test comparing means of \% $\mathrm{BF}$ from two independent groups (level of significance: 0.05 , power: $80 \%$, and anticipated lost-to-follow-up rate: $10 \%)$.

\section{Statistical methods}

This trial recorded core data on nutrition, as recommended by consensus groups. ${ }^{18}$ Descriptive data were expressed as means (SD) or numbers (percentages) of observations. For analysis of the primary outcome, an unadjusted $t$-test comparison of the mean \%BF between groups was planned. However, due to unexpected missing data for the primary outcome, a repeated-measures mixed model was performed using longitudinal \%BF data from 30 to 32 weeks PMA to 3 months CA. For this analysis that accounted for subject-to-subject variability (random effects) and a fixed interaction term between the study groups and time (fixed effects), randomness in missing data and unstructured correlations were assumed. ${ }^{20} \mathrm{~A}$ similar approach was used for analyses of secondary outcomes. All statistical analyses were performed using JMP Pro, version 14 (SAS Institute Inc., Cary, NC).

\section{RESULTS}

Of the 143 extremely preterm infants $25-28$ weeks of gestation born during the study period, 56 were randomized (Fig. 1). The median gestational age of these infants was 27 weeks (IQR: 26-28 weeks) and the mean birthweight was $957 \mathrm{~g}$ (SD: $260 \mathrm{~g}$ ). Of the 56 infants included, 50 had at least one body composition measurement after randomization (88\%). Body composition was measured in 17 infants at 30-32 weeks PMA, in 46 infants at 36 weeks PMA, and in 23 infants at 3 months CA. Due to respiratory support requirement, many infants did not have inpatient body composition measurements at 30-32 weeks PMA. Of the 6 infants on respiratory support at 36 weeks PMA, 3 remained on respiratory support at 3 months CA.

The baseline characteristics of the study participants did not differ between groups (Table 1). Randomization and treatment allocation occurred at $\sim 2$ weeks after birth. The average duration of the study intervention was 23 days. The average enteral protein intake after randomization was $3.9 \pm 1.5 \mathrm{~g} / \mathrm{kg} /$ day in the high protein group and $3.3 \pm 1.2 \mathrm{~g} / \mathrm{kg} /$ day in the standard protein group $(p=0.004)$. The protein/energy ratios were also higher in the high protein group (Fig. 2). Significant differences in enteral energy intake between groups were not found $(117 \pm 34$ vs. $113 \pm 33 \mathrm{kcal} / \mathrm{kg} / \mathrm{day} ; p=0.35)$ (Fig. 2).

Fifty infants were included in the primary outcome analysis. The repeated-measures mixed model included 86 body composition measurements. It revealed that the mean \%BF values did not differ over time between groups ( $17 \pm 3$ vs. $15 \pm 4 ; p=0.09$ ) (Fig. 3a). The longitudinal analysis of FFM data from 30 to 32 weeks PMA to 3 months CA showed that FFM $z$ score values were higher in the high protein group, but this difference did not reach statistical significance (mean difference favoring the high protein group: +0.6 points; $95 \% \mathrm{Cl}-0.1$ to $1.4 ; p=0.07$ ) (Fig. 3c). The longitudinal analyses of anthropometric data from birth to 3 months CA demonstrated that weight and length $z$ score values were higher in the high protein group (Fig. 3d, e).

Growth and body composition outcomes at 36 weeks PMA are shown in Table 2. FFM and FM $z$ scores at 36 weeks PMA were 


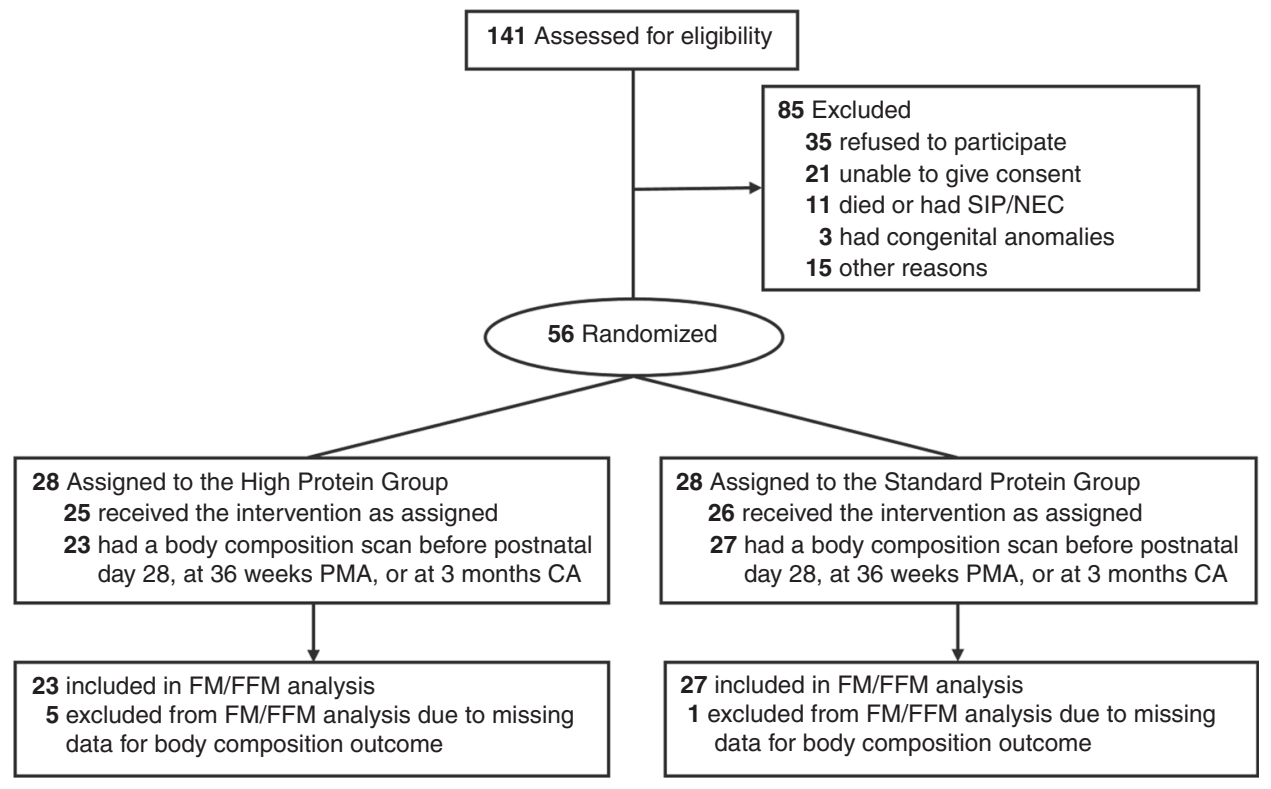

Fig. 1 Participant flow diagram. Enrollment, randomization, and outcomes.

Table 1. Baseline characteristics.

\begin{tabular}{|c|c|c|}
\hline & Intervention group $(n=28)$ & Control group $(n=28)$ \\
\hline \multicolumn{3}{|l|}{ Demographic characteristics } \\
\hline Birthweight in grams, median (IQR) & $940 \pm 307$ & $982 \pm 272$ \\
\hline Weight-for-age $z$ score at birth, mean $\pm S D^{a}$ & $0 \pm 1.7$ & $-0.1 \pm 1.3$ \\
\hline Weight $<10$ th percentile at birth, $(\%)$ & $5 / 28(18)$ & $5 / 28(18)$ \\
\hline Male, $\mathrm{n}(\%)$ & $10 / 28(36)$ & $14 / 28(50)$ \\
\hline Exposure to a full course ( 2 doses) of antenatal steroids, $n(\%)$ & $21 / 28(75)$ & $21 / 28(75)$ \\
\hline Vaginal delivery, $n(\%)$ & $22 / 28(79)$ & $24 / 28(86)$ \\
\hline Multiple gestations, $n(\%)$ & $11 / 28(39)$ & $8 / 28(29)$ \\
\hline Postnatal age when consent was obtained in days, median (IQR) & $9(3-13)$ & $8(4-13)$ \\
\hline Postnatal age when enteral feeding was initiated in days, median (IQR) & $3(2-4)$ & $3(3-4)$ \\
\hline Parenteral protein intake in $\mathrm{g} / \mathrm{kg}$ at postnatal day 7 , mean \pm SD & $3.3 \pm 1.0$ & $3.4 \pm 1.4$ \\
\hline Duration of parenteral nutrition in days, median (IQR) & $5(3-7)$ & $5(2-9)$ \\
\hline
\end{tabular}

$\mathrm{a}_{z}$ scores were estimated with the INTEGROWTH-21st growth curves.

higher in the intervention group. Growth rates and declines in $z$ scores from birth to 36 weeks PMA and from postnatal days 7-36 weeks PMA did not differ significantly between groups. The median length of hospital stay was 75 days (IQR: 64-104). During the first 28 days after birth, five infants had BUN values $>40 \mathrm{mg} / \mathrm{dl}$ ( 3 in the high protein group and 2 in the standard protein group) and seven infants had total serum $\mathrm{CO}_{2}$ values of $16 \mathrm{mmol} / \mathrm{l}$ or less (4 in the high protein group and 3 in the standard protein group). The median BUN values did not differ between the high and the standard protein group ( $26 \pm 9$ vs. $23 \pm 10 \mathrm{mg} / \mathrm{dl}$, respectively). Serum concentrations of albumin and ammonia were not routinely measured.

Pearson correlation coefficients of a post hoc correlation analysis between energy/protein intake and anthropometric/body composition measurements at 36 weeks PMA are shown in Table 3. P/E ratio at postnatal day 21 had the strongest correlation with weight and FFM $z$ scores at 36 weeks PMA.

From birth to 120 days, none of the infants included in the trial died or developed NEC stage 2 or greater. Three infants received postnatal steroids ( 2 in the high protein group and 1 in the standard protein group). Between high and standard protein groups, there were no differences in the duration of mechanical ventilation $(5 \pm 8$ vs. $3 \pm 4)$, the duration of respiratory support with continuous positive airway pressure $(13 \pm 8$ vs. $13 \pm 8)$, or the number of days on supplemental oxygen during the first 28 days after birth ( $20 \pm 10$ vs. $18 \pm 10)$. There were no serious events related to body composition measurements. 
Energy Intake

$p=0.35$

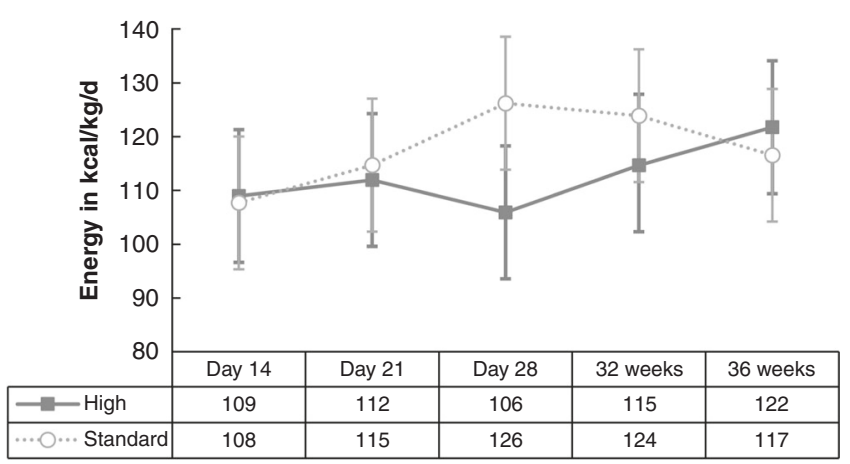

b
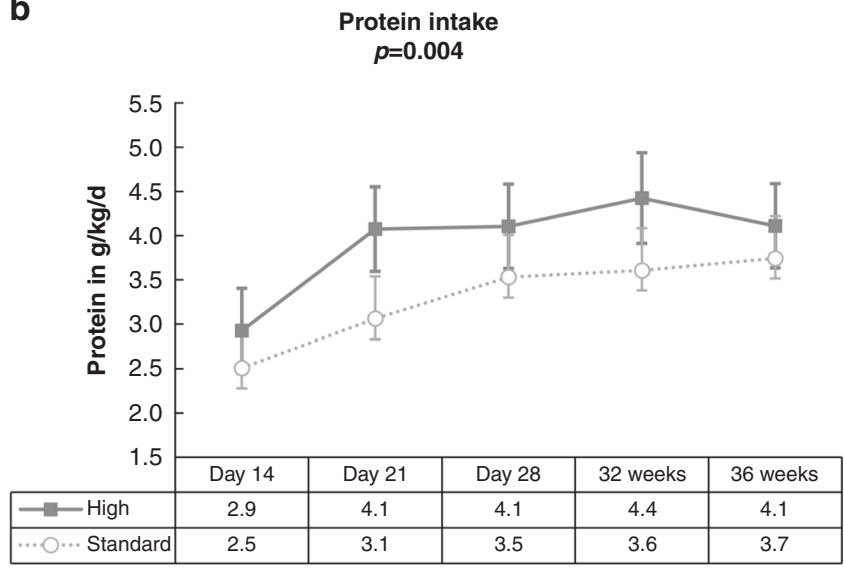

C

Protein/energy ratio $p<0.0001$

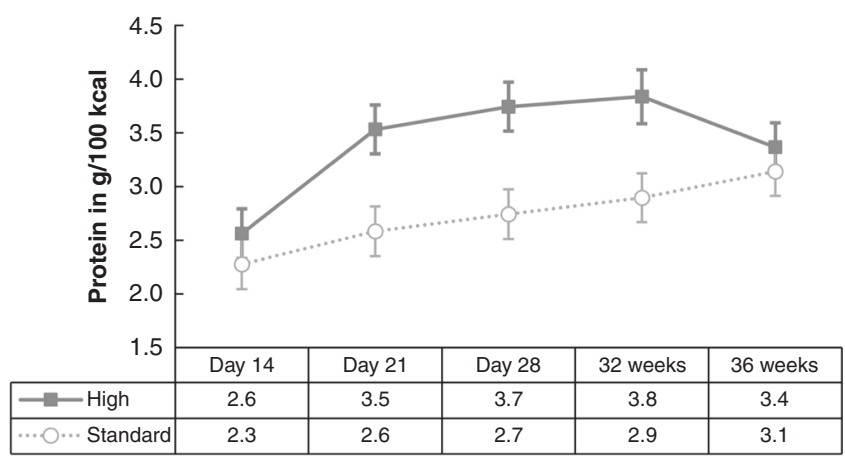

Fig. 2 Energy and protein intake according to intervention group. Longituinal changes in energy intake (a), protein intake (b), and protein/energy ratios (c). Mean values and $95 \% \mathrm{Cls}$ were adjusted with repeated measures analyses that assumed an unstructured variance and accounted for subject-to-subject variability (random effects) and a fixed interaction term between the study groups and time (fixed effects).

\section{DISCUSSION}

This single-center, masked, randomized trial examined the effects of high protein supplementation on body composition outcomes of extremely preterm infants fed fortified human milk. This supplementation method did not result in lower \%BF at any of the three time points analyzed (30-32 weeks PMA, 36 weeks PMA, or 3 months (A). However, FM and FFM $z$ scores at 36 weeks PMA, $\sim 4$ weeks after the intervention was discontinued per study protocol, increased more in the supplemented group. A repeated- measures analysis of longitudinal FFM $z$ score data revealed that the effect of higher enteral protein intake on higher FFM found at 36 weeks PMA was also evident at 30-32 weeks PMA and 3 months CA, but these early and long-lasting effects did not reach statistical significance. In contrast, a similar analysis of longitudinal FM $z$ score data showed that the effect of higher enteral protein intake on higher FM found at 36 weeks PMA was transient. After increasing the enteral intake of protein for $\sim 3$ weeks, we did not observe an increased risk of metabolic acidosis and uremia.

Adding a fixed amount of protein to fortified human milk resulted in higher $P / E$ ratios and higher FFM gains at 36 weeks PMA, but it did not significantly affect growth rates or changes in weight $z$ scores over time. Because the total energy intake did not differ between groups, we did not anticipate differences in growth rates or weight $z$ score changes from birth to 36 weeks PMA. When infants receive human milk, actual enteral protein intake is often lower than assumed enteral protein intake. We proposed this pragmatic supplementation approach to correct the $0.5-0.8 \mathrm{~g} /$ $\mathrm{kg} /$ day deficit of protein reported in extremely preterm infants fed powdered human milk fortifiers. ${ }^{21}$ Another pragmatic approach that could correct this deficit is increasing the current industry standard of protein content in commercially available liquid human milk fortifiers.

Our results support the claims that higher protein/energy ratios increase FFM accretion in preterm infants ${ }^{22}$ and that FFM gains resulting from higher enteral protein intake are associated with improved growth in preterm infants fed human milk. ${ }^{23,24}$ This effect on FFM has potential long-term benefits because several observational studies have shown that higher FFM gains are associated with a lower risk of adverse neurodevelopmental outcomes. ${ }^{8,25-27}$

By selecting \%BF as the primary outcome of this trial, we expected that increased enteral protein intake would result in lower \%BF and higher FFM accretion. However, recent studies suggest that early changes in \%BF from birth to 36 weeks are an adaptive response to the extrauterine environment independent of nutrient intake ${ }^{28}$ and that lower $\% \mathrm{BF} z$ scores do not correlate with higher FFM $z$ scores. ${ }^{29}$

One of the main limitations of this trial was the decreased power for several of the comparisons reported. Due to an unexpected pause in research activities that included the cancellation of outpatient visits, we had to replace our original aim. With a repeated-measures analysis that allows comparisons of overall mean values between groups, we maximized the value of the 86 body composition measurements obtained during the trial and addressed the problem of randomness in missing data.

Another important limitation was the reduced number of inpatient body composition measurements at 30-32 weeks PMA. We systematically excluded extremely preterm infants 22-24 weeks of gestation to minimize this limitation. However, this approach was insufficient to have a baseline measurement of infant body composition. By the time extremely preterm infants were stable enough to have their body composition measurement with ADP at 30-32 weeks PMA, most of them were already receiving the study intervention. We have demonstrated the feasibility of obtaining baseline measurements soon after birth in infants born at $28-32$ weeks. ${ }^{29}$ However, the results of this trial suggest that this might not be possible in more immature extremely preterm infants. Other limitations were the singlecenter study design, the calculations used to assume enteral protein intake without a human milk analyzer, and the lack of serum measurements of amino acid concentrations after protein supplementation.

The main strengths of this study are randomization, masking, and ADP measurements of body composition in extremely preterm infants. Other trials of protein supplementation did not include body composition as an outcome. ${ }^{4}$ ADP is a non- 


$$
\% \text { BF }
$$

$p=0.09$



b

$$
\text { Fat mass }
$$

$p=0.09$

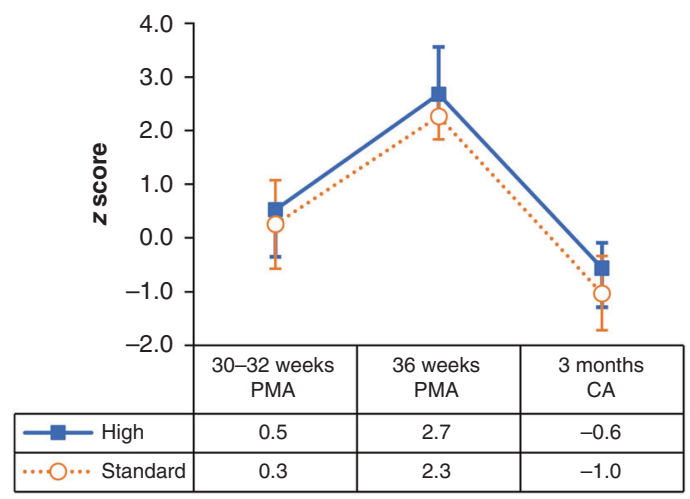

\section{C}

Fat free mass $p=0.07$

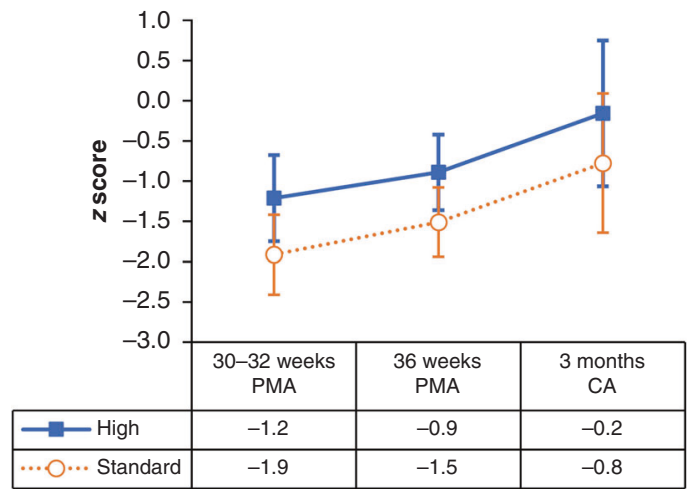

d

Weight $p=0.02$

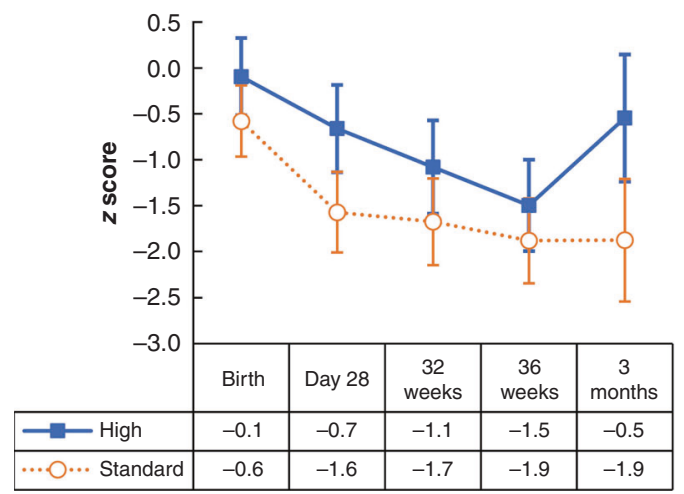

e

Length

$p=0.03$

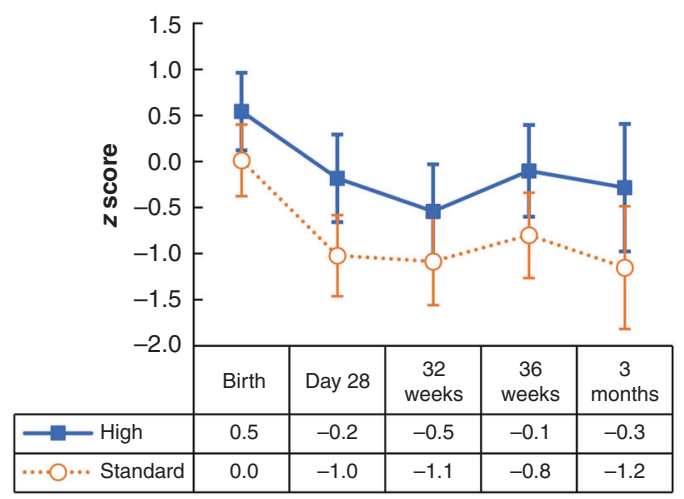

f $p=0.31$

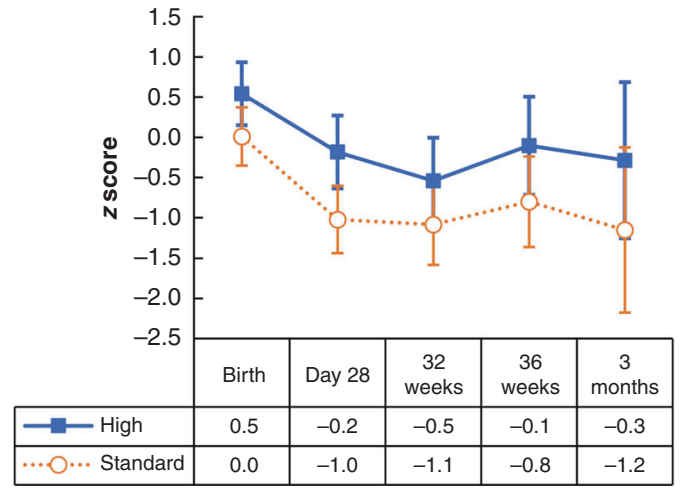

Fig. 3 Growth and body composition outcomes from birth to 3 months of corrected age according to intervention group. Longituinal changes in body fat percentage (a), fat mass (b), fat-free mass (c), weight (d), length (e), and head circumference (f). Mean values and $95 \%$ Cls were adjusted with repeated measures analyses that assumed an unstructured variance and accounted for subject-to-subject variability (random effects) and a fixed interaction term between the study groups and time (fixed effects).

invasive and accurate method to measure body composition. ${ }^{30}$ Other strengths include the reporting on early infancy outcomes in a neonatal trial, the report of growth outcomes as recommended by consensus groups, and the use of the intention-to-treat principle to preserve the effect of randomization.

Providing information on body composition outcomes during early infancy is a high-priority field of nutrition research. Ideally, future multicenter trials should include only high-risk, immature, extremely preterm infants (mainly 22-24 weeks of gestation) and more extended periods of protein supplementation. FFM $z$ scores should be the primary outcome of future trials. Because body composition measurements at 3 months of age in term infants may not be the ideal reference for preterm infants, these trials should consider using or developing new reference values generated with longitudinal data of body composition measurements in preterm infants. Future multicenter studies with larger sample sizes should also investigate alternative methods to assess 
Table 2. Study outcomes in extremely preterm infants with body composition measurements at 36 weeks PMA or hospital discharge.

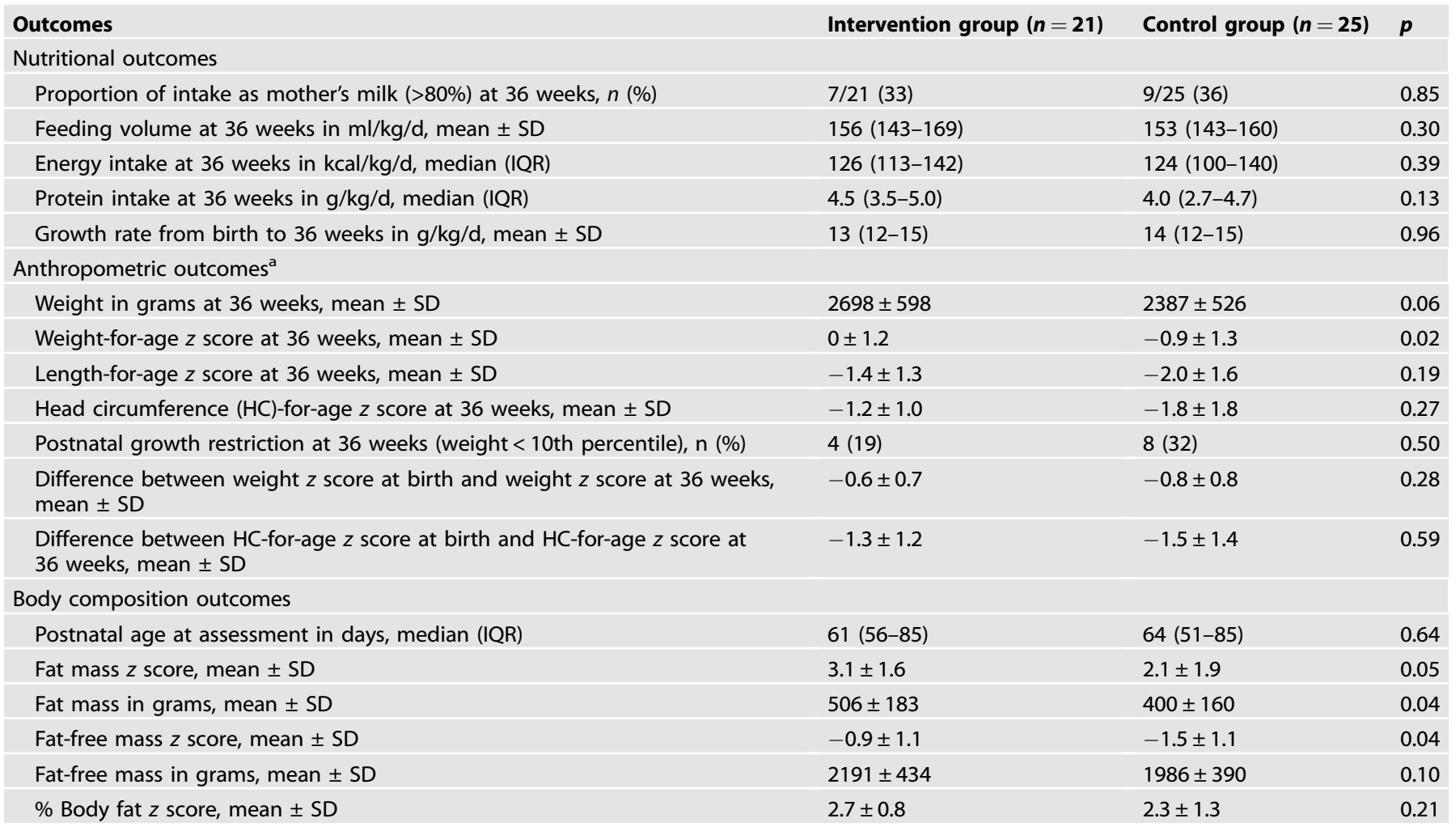

$\mathrm{a}_{z}$ scores were estimated with the INTEGROWTH-21st growth curves.

Table 3. Pearson coefficients of correlation analyses between enteral intake at postnatal days 21-28 and growth outcomes at 36 weeks PMA.

\begin{tabular}{|c|c|c|c|c|}
\hline & \multicolumn{4}{|c|}{$z$ score outcomes at 36 weeks PMA ${ }^{a}$} \\
\hline & Weight & Length & Head circumference & Fat-free mass \\
\hline \multicolumn{5}{|l|}{ Enteral intake } \\
\hline Protein intake at day 21 & 0.45 & 0.30 & 0.25 & 0.29 \\
\hline Protein/energy ratio at day 21 & 0.50 & 0.34 & 0.28 & 0.42 \\
\hline Caloric intake at day 28 & 0.23 & 0.02 & 0.12 & 0.04 \\
\hline Protein/energy ratio at day 28 & 0.54 & 0.34 & 0.37 & 0.35 \\
\hline
\end{tabular}

a'Weight, length, and head circumference $z$ scores were estimated with the INTEGROWTH-21st growth curves.

FFM accretion. ADP is not always feasible in extremely preterm infants.

In conclusion, supplementation with extensively hydrolyzed protein in extremely preterm infants fed multi-nutrient fortified human milk did not decrease \%BF. However, this supplementation method increased weight, length, and FFM $z$ scores from 30 to 32 weeks PMA to 3 months CA in extremely preterm infants. Larger trials are needed before implementing high protein supplementation in extremely preterm infants.

\section{REFERENCES}

1. Travers, C. P. et al. Racial/ethnic disparities among extremely preterm infants in the United States From 2002 to 2016. JAMA Netw. Open 3, e206757 (2020).

2. Stoll, B. J. et al. Trends in care practices, morbidity, and mortality of extremely preterm neonates, 1993-2012. JAMA 314, 1039-1051 (2015).
3. Brown, J. V., Embleton, N. D., Harding, J. E. \& McGuire, W. Multi-nutrient fortification of human milk for preterm infants. Cochrane Database Syst. Rev. 5, CD000343 (2016).

4. Amissah, E. A., Brown, J. \& Harding, J. E. Protein supplementation of human milk for promoting growth in preterm infants. Cochrane Database Syst. Rev. 6, CD000433 (2018).

5. Reid, J. et al. The effect of increasing the protein content of human milk fortifier to $1.8 \mathrm{~g} / 100 \mathrm{~mL}$ on growth in preterm infants: a randomised controlled trial. Nutrients 10, 634 (2018).

6. Maas, C. et al. Effect of increased enteral protein intake on growth in human milkfed preterm infants: a randomized clinical trial. JAMA Pediatr. 171, 16-22 (2017).

7. Bell, K. A. et al. Associations of growth and body composition with brain size in preterm infants. J. Pediatr. 214, 20-26 (2019).

8. Ramel, S. E. et al. Greater early gains in fat-free mass, but not fat mass, are associated with improved neurodevelopment at 1 year corrected age for prematurity in very low birth weight preterm infants. J. Pediatr. 173, 108-115 (2016). 
9. Ong, K. K. et al. Postnatal growth in preterm infants and later health outcomes: a systematic review. Acta Paediatr. 104, 974-986 (2015).

10. Morrison, K. M. et al. Cardiometabolic health in adults born premature with extremely low birth weight. Pediatrics 138, e20160515 (2016).

11. Raju, T. N. K., Buist, A. S., Blaisdell, C. J., Moxey-Mims, M. \& Saigal, S. Adults born preterm: a review of general health and system-specific outcomes. Acta Paediatr. 106, 1409-1437 (2017).

12. Cordova, E. G. \& Belfort, M. B. Updates on assessment and monitoring of the postnatal growth of preterm infants. Neoreviews 21, e98-e108 (2020).

13. Belfort, M. B. \& Ramel, S. E. NICU diet, physical growth and nutrient accretion, and preterm infant brain development. Neoreviews 20, e385-e396 (2019).

14. Norris, T. et al. New charts for the assessment of body composition, according to air-displacement plethysmography, at birth and across the first 6 mo of life. Am. J. Clin. Nutr. 109, 1353-1360 (2019).

15. Hay, W. W. Jr. Optimizing protein intake in preterm infants. J. Perinatol. 29, 465-466 (2009).

16. Raiten, D. J. et al. Working group reports: evaluation of the evidence to support practice guidelines for nutritional care of preterm infants-the Pre-B Project. Am. J. Clin. Nutr. 103, 648S-678S (2016).

17. Belfort, M. et al. Macronutrient intake from human milk, infant growth, and body composition at term equivalent age: a longitudinal study of hospitalized very preterm infants. Nutrients 12, 2249 (2020).

18. Cormack, B. E., Embleton, N. D., van Goudoever, J. B., Hay, W. W. Jr. \& Bloomfield, F. H. Comparing apples with apples: it is time for standardized reporting of neonatal nutrition and growth studies. Pediatr. Res. 79, 810-820 (2016).

19. Villar, J. et al. Postnatal growth standards for preterm infants: the Preterm Postnatal Follow-up Study of the INTERGROWTH-21(st) Project. Lancet Glob. Health 3, e681-e691 (2015).

20. Detry, M. A. \& Ma, Y. Analyzing repeated measurements using mixed models. JAMA 315, 407-408 (2016).

21. Arslanoglu, S., Moro, G. E. \& Ziegler, E. E. Preterm infants fed fortified human milk receive less protein than they need. J. Perinatol. 29, 489-492 (2009).

22. Lingwood, B. E., Al-Theyab, N., Eiby, Y. A., Colditz, P. B. \& Donovan, T. J. Body composition in very preterm infants before discharge is associated with macronutrient intake. Br. J. Nutr. 123, 800-806 (2020).

23. Morlacchi, L. et al. Protein use and weight-gain quality in very-low-birth-weight preterm infants fed human milk or formula. Am. J. Clin. Nutr. 107, 195-200 (2018).

24. Bruckner, M. et al. Extremely preterm infants have a higher fat mass percentage in comparison to very preterm infants at term-equivalent age. Front Pediatr. 8, 61 (2020).

25. Pfister, K. M. et al. Early body composition changes are associated with neurodevelopmental and metabolic outcomes at 4 years of age in very preterm infants. Pediatr. Res. 84, 713-718 (2018).

26. Frondas-Chauty, A. et al. Deficit of fat free mass in very preterm infants at discharge is associated with neurological impairment at age 2 years. J. Pediatr. 196, 301-304 (2018).

27. Scheurer, J. M. et al. Body composition trajectories from infancy to preschool in children born premature versus full-term. J. Pediatr. Gastroenterol. Nutr. 64, e147-e153 (2017).

28. Simon, L. et al. Determinants of body composition in preterm infants at the time of hospital discharge. Am. J. Clin. Nutr. 100, 98-104 (2014).
29. Salas, A. A., Jerome, M. L., Chandler-Laney, P., Ambalavanan, N. \& Carlo, W. A Serial assessment of fat and fat-free mass accretion in very preterm infants: a randomized trial. Pediatr. Res. 88, 733-738 (2020).

30. Ellis, K. J. et al. Body-composition assessment in infancy: air-displacement plethysmography compared with a reference 4-compartment model. Am. J. Clin. Nutr. 85, 90-95 (2007).

\section{ACKNOWLEDGEMENTS}

This trial was supported by a research grant from the Kaul Pediatric Research Institute of the Children's Hospital of Alabama. A.A.S. is currently supported by a research grant from the Eunice Kennedy Shriver National Institute of Child Health and Human Development (K23HD102554)

\section{AUTHOR CONTRIBUTIONS}

The authors' responsibilities were as follows-A.A.S. conceptualized and designed the study, supervised data collection, performed the statistical analyses, drafted the initial manuscript, and reviewed and revised the manuscript. M.J. designed data collection instruments, performed body composition measurements, and completed follow-up visits. A.F. assisted with random allocation procedures, monitored patient enrollment and compliance, and collected nutritional data. J.R. curated and analyzed daily nutritional data. P.C.-L. assisted with the study design and critically reviewed the manuscript for important intellectual content. W.C. assisted with the study design and critically reviewed the manuscript for important intellectual content.

\section{COMPETING INTERESTS}

P.C.-L. and A.A.S. have filed a patent application for an instrumented feeding bottle. A.A.S. has received honoraria from the Lockwood Group for participation in Mead Johnson Nutrition advisory board meetings. W.A.C. is on the board of directors of Mednax, Inc. M.J., A.F., and J.R. have no financial conflicts to disclose. The protein supplement used in this study was provided free of charge by Abbott. Abbott had no role in the design of the study, collection, analysis, interpretation of data, or writing of the manuscript.

\section{CONSENT STATEMENT}

Patient consent was required for this clinical trial.

\section{ADDITIONAL INFORMATION}

Correspondence and requests for materials should be addressed to A.A.S.

Reprints and permission information is available at http://www.nature.com/ reprints

Publisher's note Springer Nature remains neutral with regard to jurisdictional claims in published maps and institutional affiliations. 